\title{
平面单测点法评价湿度和温度对药物稳定性的影响
}

\author{
蒙明程李琳丽* 詹先成 陶建林韩 雪 \\ (四川大学华西药学院, 靶向药物与释药系统教育部重点实验室, 成都 610041)
}

\begin{abstract}
摘要: 利用均匀设计分散原理, 确定药物的湿度温度平面, 采用单测点法获得药物相关的降解动力学参数. 阿 司匹林稳定性加速试验结果表明, 平面单测点法与恒温恒湿法和程序变温变湿法得到的降解动力学参数基本一 致. 在药物降解程度及试验的湿度差和温度差相同的条件下, 平面单测点法结果的准确度和精密度均明显优于 程序变温变湿法, 且装置简单. 与经典恒温恒湿法相比, 平面单测点法的误差较大, 但试验工作量减少.
\end{abstract}

关键词： 平面单测点法； 恒温恒湿法； 程序变温变湿法；模拟试验；阿司匹林 中图分类号: $\mathrm{O} 643$

\section{Evaluation of the Influence of Humidity and Temperature on the Drug Stability by Plane Single Time Point Experiments}

\author{
MENG Ming-Cheng LI Lin-Li ${ }^{*}$ ZHAN Xian-Cheng TAO Jian-Lin HAN Xue \\ (Key Laboratory of Drug Targeting and Drug Delivery System of Ministry of Education, West China School of Pharmacy, \\ Sichuan University, Chengdu 610041, P. R. China)
}

\begin{abstract}
The moisture and temperature plane of a drug was determined based on the dispersive principle of uniform design. Then, kinetic parameters were obtained by a single time point experiment. The stability of aspirin, as a solid state model, was investigated. We found that the results obtained from this proposed method were comparable to those from isothermal studies at constant humidity and also comparable to those from the programmed humidifying and heating experiments. Under the same experimental conditions, the estimates of the kinetic parameters $\left(E_{\mathrm{a}}, m, A\right)$ obtained from the new method were significantly more accurate and precise than those obtained from the programmed humidifying and heating experiments. No specific computer-controlled environmental chamber needs to be used in our new method. By comparison to isothermal experiments at constant humidity, our proposed method saved time, labor, and materials.
\end{abstract}

Key Words : Plane single time point experiments; Classical isothermal experiment at constant humidity; Programmed humidifying and heating experiment; Simulated test; Aspirin

研究湿度和温度对固体药物稳定性综合影响 的方法有经典恒温恒湿 ${ }^{[1-8]}$ 法和程序变温变湿加速 实验 ${ }^{[9-11]}$ 法. 前者准确度高, 但耗时长; 后者在特制的 程序变湿变温装置中, 采用一次恒温条件下程序变 湿加速实验和一次恒湿条件下程序变温加速试验, 得到了相关的降解动力学参数. 有人曾提出台阶变 温变湿加速实验 ${ }^{[12]}$, 通过人工逐次改变实验湿度和
温度, 从而可利用普通的恒温恒湿箱来控制湿度和 温度. 程序变温变湿与台阶变温变湿这两种简化的 加速试验方法, 均是采用单因素的分类试验方法, 即固定某一影响因素, 如温度(或湿度), 让另一因素 湿度(或温度)按一定的规律改变, 分两步实现药物 稳定性加速试验. 在以上简化的加速试验法中, 动 力学参数是通过两类实验的结果取平均值求得的,

Received: December 9, 2008; Revised: February 16, 2009; Published on Web: February 26, 2009.

*Corresponding author. Email: ysylilinli@sina.com; Tel: +8628-85501385.

国家自然科学基金(20872100)资助项目

(C) Editorial office of Acta Physico-Chimica Sinica 
假定湿度和温度对药物稳定性的影响权重相同. 但 对大多数药物而言, 湿度和温度的影响程度是不相 同的, 有的是对热敏感, 有的是湿度对其稳定性的影 响更大. 因此, 这两种简化加速试验法结果的准确度 及精密度均低.

庞贻慧等 ${ }^{[13-15]}$ 曾采用单测点法代替恒温加速试 验, 只需测定反应进行到某个时刻药物的含量, 然后 结合初始含量数据就可求得该温度下药物的降解速 率常数 $k$. 由于在每个温度下只测定一个含量数据, 所求得的 $k$ 值误差较大, 作为补偿, 在单测点法中将 试验温度数增加. 若将单测点法的原理用于研究药 物对湿度的稳定性, 也可能具有减少试验次数的优 点.

本文提出的平面单测点法是在选取几个湿度、 温度水平基础上, 利用均匀设计 ${ }^{[16-23]}$ 组合所选的湿 度和温度, 再采用单测点法在湿度和温度的最佳组 合下进行药物稳定性加速试验, 求出药物的降解动 力学参数. 该新方法不仅所需试验工作量小、准确度 高, 而且避免了通过分类试验结果取平均值求动力 学参数的缺陷. 通过研究湿度和温度对固体药物阿 司匹林的降解, 介绍了平面单测点法研究化学稳定 性的方法.

\section{1 理论部分}

若药物对湿度和温度均不稳定, 其降解速率常 数 $k$ 与温度和湿度的关系可表达为 ${ }^{[3,7,8]}$.

$$
\ln k=\ln A+m H_{\mathrm{r}}-\frac{E_{\mathrm{a}}}{R T}
$$

其中 $H_{\mathrm{r}}$ 为相对湿度, $T$ 为热力学温度, $R$ 为气体常 数, $A$ 为指前因子, $E_{\mathrm{a}}$ 为活化能, $m$ 为一个只决定于 药物的本性而与湿度和温度均无关的常数.

$1 / T, H_{\mathrm{r}}$ 和 $\ln k$ 可构成三维空间中的一个湿度温 度平面(如图 1 所示). 理论上, 通过三个湿度温度水 平组合点 $\left(1 / T_{i}, H_{\mathrm{r}}, \ln k_{i}\right)$, 只要这三个点不在一条直线 上, 就可以确定该湿度温度平面.

为了能精确地确定湿度、温度平面, 同时使试 验工作量最少, 本文采用均匀设计的分散原理 ${ }^{[16]}$, 寻 求湿度与温度的最佳组合, 然后在每个组合点下用 单测点法进行药物稳定性加速试验, 从而可获得药 物相关的降解动力学参数. 我们把这种方法叫作平 面单测点恒温恒湿法(简称平面单测点法).

例如, 在一定温度范围 $T_{1}-T_{11}$ 内均匀地选取 11 个温度水平, 在一定湿度范围 $H_{\mathrm{r} 1}-H_{\mathrm{r} 11}$ 内也均匀地

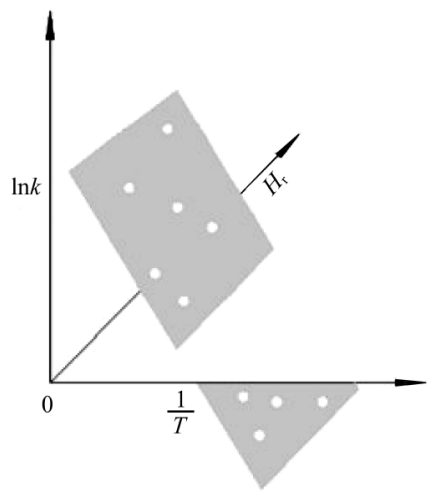

图 1 湿度温度平面三维坐标示意图

Fig.1 Schematic diagram of the moisture and temperature plane in three dimensional space

选取 11 个湿度水平. 运用均匀设计 $U_{11}^{*}\left(11^{4}\right)$ 对这些 湿度、温度水平进行处理, 寻求其最佳组合(如图 2 所示), 在这些最佳组合下用单测点法进行药物稳定 性加速试验, 然后利用最小二乘法拟合试验数据, 即 可确定该湿度温度平面, 进而求出相关的动力学 参数.

阿司匹林对湿度和温度均不稳定, 其降解不是 一个简单级数反应. 在恒温恒湿条件下, 阿司匹林的 降解在反应初期速率较慢, 中期反应速率加快, 后期 反应速率减慢. 其速率常数 $k$ 与药物浓度 $c(c$ 是药物 的实测值与样品取样量的质量之比, 单位为 $\left.g \cdot g^{-1}\right)$ 之间的关系为 ${ }^{[11]}$ :

$$
\ln \frac{c_{0}-c}{c}=k t-3.37 \quad\left(c \neq c_{0}\right)
$$

式中 $c_{0}$ 为药物的初始浓度. 将(2)式代人(1)式, 整理 后有

$$
\frac{\ln \left[\left(c_{0}-c\right) / c\right]+3.37}{t}=A \exp \left(m H_{\mathrm{r}}\right) \exp \left(-\frac{E_{\mathrm{a}}}{R T}\right)
$$

本文以阿司匹林为模型药物, 采用平面单测点

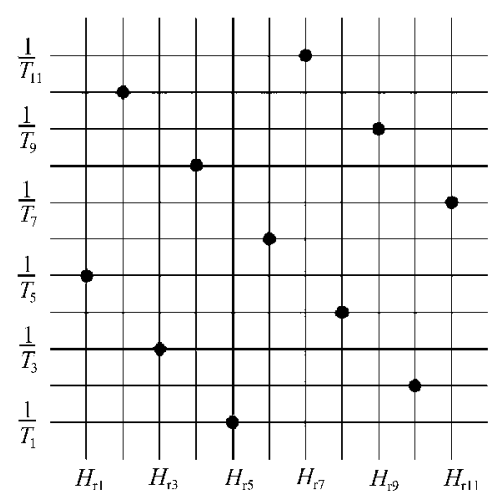

图 2 均匀设计法确定湿度温度组合

Fig.2 Decision of combination of moisture and temperature with the uniform design 
法研究湿度和温度同时对其稳定性的影响.

\section{2 实验部分}

\section{1 仪器与药品}

阿司匹林(山东新华制药厂, 含量以 $\mathrm{C}_{9} \mathrm{H}_{8} \mathrm{O}_{4}$ 计 为 $99.62 \%$ ); 其它试剂均为分析纯. SDH401 型恒温 恒湿箱(重庆银河实验仪器有限公司).

\section{2 样品的含量测定}

采用两步滴定法测定降解过程中阿司匹林含量 的变化 ${ }^{[11,24,25]}$. 取阿司匹林约 $0.15 \mathrm{~g}$ 于烧杯中, 精密 称定, 加人中性乙醇 $10 \mathrm{~mL}$ 溶解后, 以酚酞为指示 剂, 滴加 $\mathrm{NaOH}$ 溶液 $\left(0.1 \mathrm{~mol} \cdot \mathrm{L}^{-1}\right)$ 滴定至溶液显粉红 色. 此时中和了样品中原有的水解产物(水杨酸、醋 酸). 在中和后的待测样品溶液中, 精密加人 $\mathrm{NaOH}$ 滴 定液 $\left(0.1 \mathrm{~mol} \cdot \mathrm{L}^{-1}\right) 20.0 \mathrm{~mL}$, 密闭, 置水浴上加热 15 min, 使阿司匹林水解. 然后迅速放冷至室温, 用 $\mathrm{H}_{2} \mathrm{SO}_{4}$ 滴定液 $\left(0.05 \mathrm{~mol} \cdot \mathrm{L}^{-1}\right)$ 滴定剩余的氢氧化钠, 并将滴定结果用空白试验校正. 样品中阿司匹林 的含量是由水解时消耗的碱量来计算的. 每 $1 \mathrm{~mL}$ 的 $\mathrm{NaOH}\left(0.1 \mathrm{~mol} \cdot \mathrm{L}^{-1}\right)$ 滴定液相当于 $18.02 \mathrm{mg}$ 的 $\mathrm{C}_{9} \mathrm{H}_{8} \mathrm{O}_{4}$.

\section{3 平面单测点恒温恒湿稳定性试验}

在一定温度范围65.0-80.0 ${ }^{\circ} \mathrm{C}$ 内选取 11 个温度 水平 $80.0 、 78.4 、 76.9 、 75.4 、 73.8 、 72.3 、 70.8 、 69.4 、 67.9$ 、 $66.4 、 65.0{ }^{\circ} \mathrm{C}$ (等 $1 / T$ 间隔); 在一定湿度范围 $60 \%-$ $90 \%$ 内选取 11 个湿度水平 $60 \% 、 63 \% 、 66 \% 、 69 \%$ 、 $72 \% 、 75 \% 、 78 \% 、 81 \% 、 84 \% 、 87 \% 、 90 \%$ (等湿度间隔), 根据均匀设计, 得到温度与湿度的最佳组合(见表1).

每次精密称取阿司匹林 3 份, 每份约 $0.15 \mathrm{~g}$, 均 匀平铺于 $50 \mathrm{~mL}$ 烧杯内. 将烧杯置于恒温恒湿箱中, 在湿度与温度的最佳组合下进行加速试验. 当含量 下降至 80\%左右时, 取出测定阿司匹林的含量.

\section{3 结果与讨论}

\section{1 平面单测点法}

利用平面单测点法研究阿司匹林的稳定性, 结 果见表 1 . 由表 1 中不同时刻阿司匹林的含量数据, 根据式(2)求出相应的速率常数 $k$ (也列人表 1 中).

按照速率常数 $k$ 的表达式(1), 采用最小二乘法 对不同湿度和温度组合下的 $k$ 值进行拟合, 可得该 湿度平面解析式为

$$
\ln k=28.28+1.20 H_{\mathrm{r}}-\frac{95.1 \times 10^{3}}{R T}
$$

表 1 阿司匹林在平面单测点试验下的稳定性结果

Table 1 Data from the stability study of aspirin in the plane single time point experiments

\begin{tabular}{rrrcc}
\hline$T /{ }^{\circ} \mathrm{C}$ & $H_{\mathrm{r}}(\%)$ & \multicolumn{1}{c}{$t / \mathrm{h}$} & $c /\left(\mathrm{g} \cdot \mathrm{g}^{-1}\right)$ & $10^{2} \mathrm{k} / \mathrm{h}^{-1}$ \\
\hline & & 0.00 & $0.9962 \pm 0.0073^{\mathrm{a}}$ & \\
80.0 & 72.00 & 50.00 & $0.8166 \pm 0.0084$ & $3.709 \pm 0.113$ \\
78.4 & 87.00 & 54.50 & $0.7810 \pm 0.0096$ & $3.818 \pm 0.106$ \\
76.9 & 66.00 & 75.50 & $0.8054 \pm 0.0079$ & $2.555 \pm 0.068$ \\
75.4 & 81.00 & 80.00 & $0.7719 \pm 0.0122$ & $2.667 \pm 0.087$ \\
73.8 & 60.00 & 120.00 & $0.7695 \pm 0.0152$ & $1.789 \pm 0.074$ \\
72.3 & 75.00 & 96.00 & $0.8288 \pm 0.0144$ & $1.842 \pm 0.106$ \\
70.8 & 90.00 & 110.00 & $0.7753 \pm 0.0081$ & $1.922 \pm 0.043$ \\
69.4 & 69.00 & 148.50 & $0.8042 \pm 0.0130$ & $1.304 \pm 0.056$ \\
69.9 & 84.00 & 132.00 & $0.8258 \pm 0.0096$ & $1.356 \pm 0.053$ \\
66.4 & 63.00 & 190.00 & $0.8341 \pm 0.0045$ & $9.115 \pm 0.172$ \\
65.0 & 78.00 & 179.00 & $0.8418 \pm 0.0060$ & $9.348 \pm 0.257$
\end{tabular}

a) The data after " \pm " are the mean standard deviation values of three experiments.

其相关系数 $r=0.9984$ (平均值). 与表达式(1)对照, 可求 出相关的动力学参数 $E_{\mathrm{a}} 、 m$ 和 $A$ (见表 2 ). 与文献 [11] 采用经典恒温恒湿法和程序变温变湿法得到的动力 学参数相比较, 可见结果基本一致.

\section{2 模拟试验}

为了从理论上探讨所建立的平面单测点法与恒 温恒湿法以及程序变温变湿法的试验误差, 用计算 机依据式(3)模拟产生出若干组加速试验数据, 以考 察加速方式以及在平面单测点法中药物降解程度、 抽样频率、湿度范围、温度范围、平均湿度、平均温 度、湿度系统误差和温度系统误差对试验误差的影 响.

\subsection{1 加速方式对试验误差的影响}

令活化能 $E_{\mathrm{a}}=100 \mathrm{~kJ} \cdot \mathrm{mol}^{-1}, m=1.5$, 指前因子 $A=$ $2 \times 10^{12} \mathrm{~h}^{-1}$.

对于平面单测点法, 在湿度范围为 60-90\%、温 度范围为 $60-80{ }^{\circ} \mathrm{C}$ 内, 分别选取 11 个湿度和温度水

\section{表 2 阿司匹林的动力学参数在三种实验方法 下测定结果的比较}

Table 2 Comparison of kinetic parameters of aspirin with the three experiments

\begin{tabular}{cccc}
\hline Model & $E_{\mathrm{a}} /\left(\mathrm{kJ} \cdot \mathrm{mol}^{-1}\right)$ & $m$ & $10^{-12} A / \mathrm{h}^{-1}$ \\
\hline experiment $\mathrm{I}^{\mathrm{a}}$ & $94.9 \pm 0.7^{\mathrm{d}}$ & $1.20 \pm 0.02$ & $1.71 \pm 0.35$ \\
experiment $\mathrm{II}^{\mathrm{b}}$ & $93.5 \pm 2.2$ & $1.18 \pm 0.19$ & $1.09 \pm 2.04$ \\
${\text { experiment } \mathrm{III}^{\mathrm{c}}}^{\mathrm{b}}$ & $95.1 \pm 1.2$ & $1.20 \pm 0.05$ & $1.91 \pm 0.68$ \\
\hline
\end{tabular}

a) isothermal experiment at constant humidity ${ }^{[11]}$, b) programmed humidifying and heating experiment $\left.{ }^{[11]}, \mathrm{c}\right)$ plane single time point experiment (this paper), d) the data after “ \pm ” are the estimated mean standard deviation values of three experiments. 

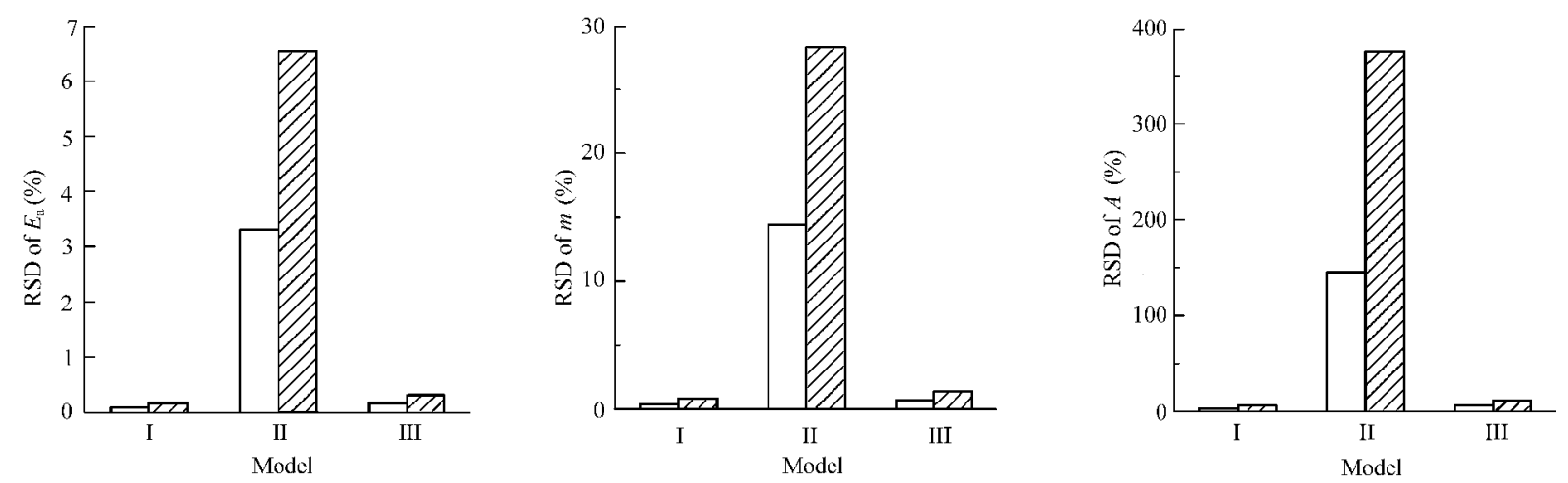

图 3 三种药物稳定性实验方法动力学参数误差比较

Fig.3 Comparison of kinetic parameter errors in three drug stability experiments

(I) isothermal experiment at constant humidity, (II) programmed humidifying and heating experiment, (III) plane single time point experiments; RSD: relative standard deviation; data with $\pm 1 \%$ ( $\square$ ) and $\pm 2 \%$ (घra) random error

平, 根据均匀设计的分散原理决定其最佳组合. 对于 程序变温变湿法, 在恒温程序变湿试验时, 令温度恒 定为 $75{ }^{\circ} \mathrm{C}$, 湿度从 $90 \%$ 按线性规律降低至 $60 \%$; 在 恒湿程序变温试验时, 令湿度恒定为 $80 \%$, 温度从 $80{ }^{\circ} \mathrm{C}$ 按倒数规律降低至 $60{ }^{\circ} \mathrm{C}$. 对于经典恒温恒湿 加速试验, 试验温度选取为 $60 、 67 、 74 、 80{ }^{\circ} \mathrm{C}$, 湿度为 $60 \% 、 70 \% 、 80 \% 、 90 \%$. 每种加速方式的任意一条稳 定性曲线均取 11 个含量数据点, 且上述三类试验的 反应物含量 $c$ 均从 $100 \%$ 降为 50\%. 依据式(3)或其 积分形式 ${ }^{[11]}$ (积分形式仅用于程序变温变湿法)分别 模拟产生出以上三种加速法的 $c-t$ 的数据, 然后分 别处理这三种加速法的数据, 结果 $\left(E_{\mathrm{a}}, m\right.$ 和 $\left.A\right)$ 与其 设定值完全一致.

为了模拟试验误差, 另在上述各种加速法 $c-t$ 数据的含量 $c$ 中, 用计算机分别叠加 $\pm 1 \%$ 和 $\pm 2 \%$ 的 随机误差, 各产生 500 组数据. 分别处理这三种加速 法的各 500 组模拟试验数据, 所得结果的相对标准 差(RSD) 见图 3.
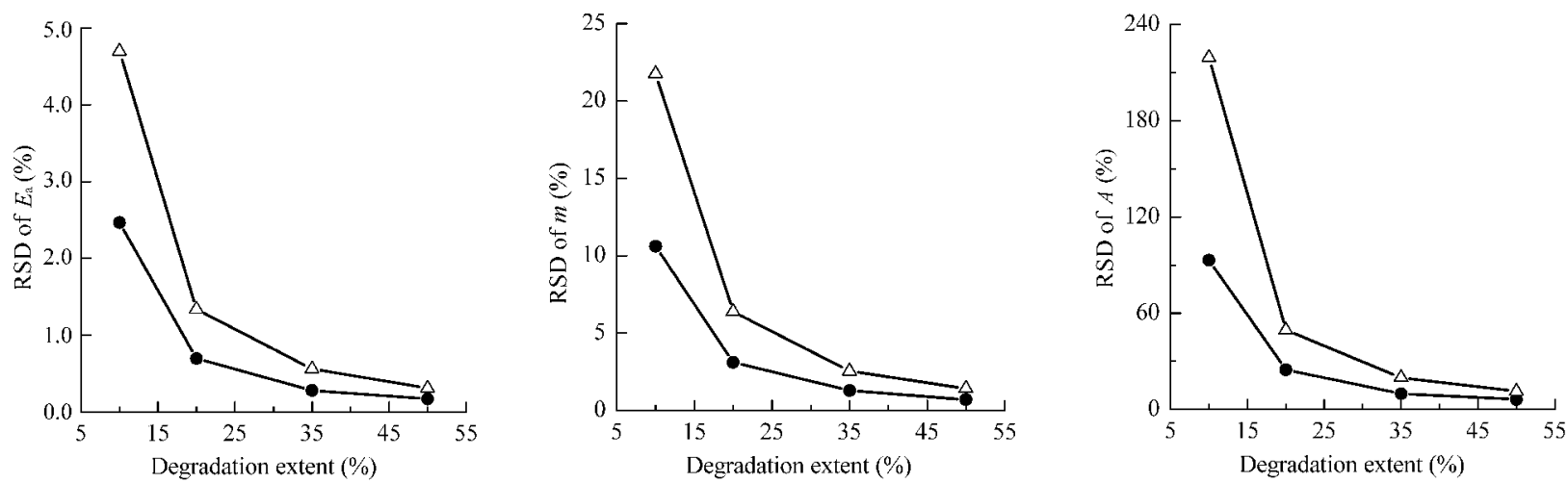

图 4 药物降解程度对动力学参数误差的影响

Fig.4 Effect of drug degradation extent on kinetic parameter errors

data with $\pm 1 \%(\bullet)$ and $\pm 2 \%(\triangle)$ random error 

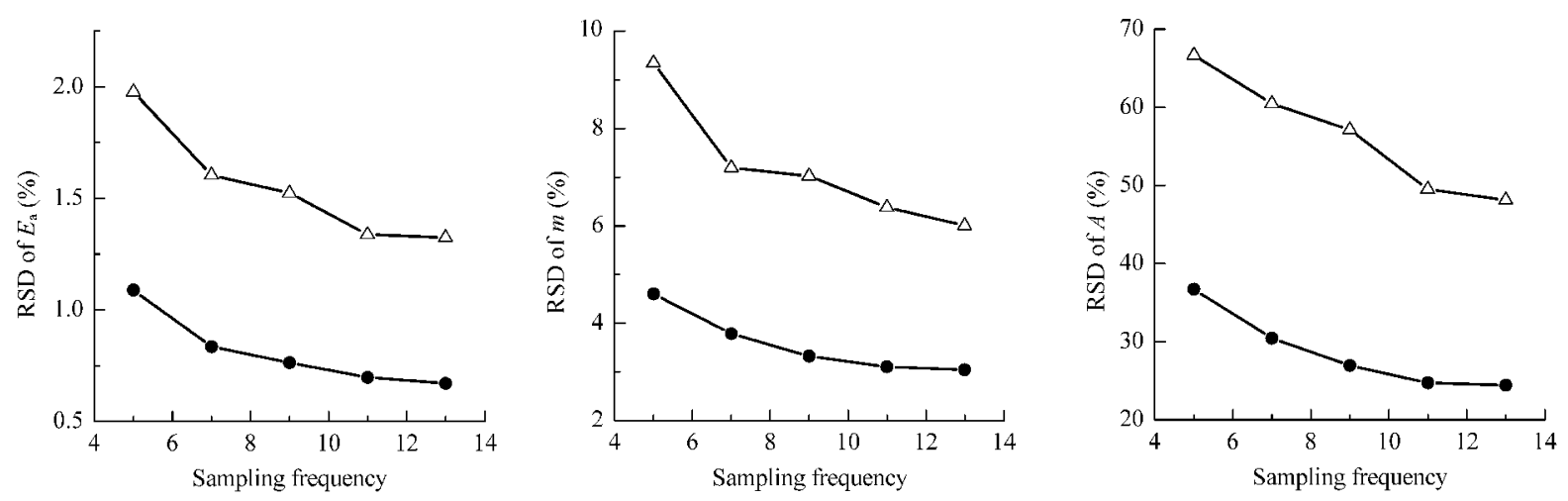

图 5 抽样频率对动力学参数误差的影响

Fig.5 Effect of sampling frequency on kinetic parameter errors

data with $\pm 1 \%(\bullet)$ and $\pm 2 \%(\triangle)$ random error

100\%降解到 80\%外, 其他条件及试验方法与 3.2 .1 节相同, 结果见图 5. 可以看出, 抽样频率越高, 试验 误差越小. 当抽样频率大于 11 时, 各动力学参数的 试验误差没有明显变化. 因此在平面单测点法中, 为 达到试验误差且工作量小的目的, 湿度与温度的最 佳组合数为 11 , 即可符合试验要求.

\subsection{4 湿度差对试验误差的影响}

除湿度范围分别为 70\%-90\%、50\%-90\%和 40\%-90\%, 含量均从 100\%降解到 80\%外, 其他条件 及试验方法与 3.2.1 节相同, 结果见图 6. 可以看出, 随着湿度差的增大, $m$ 的试验误差不断减小, 而 $E_{\mathrm{a}}$ 和 $A$ 的试验误差变化不明显.

\subsection{5 温度差对试验误差的影响}

除温度范围分别为 $70-80{ }^{\circ} \mathrm{C} 、 50-80{ }^{\circ} \mathrm{C}$ 和 40-80 ${ }^{\circ} \mathrm{C}$, 含量均从 $100 \%$ 降解到 $80 \%$ 外, 其他条件及试验 方法与 3.2.1 节相同, 结果见图 7. 可以看出, 随着温 度差的增大, $E_{\mathrm{a}}$ 和 $A$ 的试验误差不断减小, 而 $m$ 的 试验误差没有明显变化.

\subsection{6 平均湿度对试验误差的影响}

当湿度差相同, 除湿度范围分别为 65\%-95\%、 $55 \%-85 \%$ 和 $50 \%-80 \%$ (即平均湿度分别为 $80 \%$ 、 $70 \%$ 和 $65 \%$ ), 含量均从 $100 \%$ 降解到 80\%外, 其他 条件及试验方法与 3.2.1 节相同, 结果见图 8. 可以 看出: 平均湿度对各动力学参数的试验误差没有明 显影响.

\subsection{7 平均温度对试验误差的影响}

当温度差相同, 除温度范围分别为 $70-90{ }^{\circ} \mathrm{C}$, $50-70{ }^{\circ} \mathrm{C}$ 和 $40-60{ }^{\circ} \mathrm{C}$ (即平均温度分别为 $80 、 60$ 和 $50{ }^{\circ} \mathrm{C}$ ), 含量均从 $100 \%$ 降解到 $80 \%$ 外, 其他条件及 试验方法与 3.2.1 节相同, 结果见图 9. 可以看出, 平 均温度越接近室温, $E_{\mathrm{a}}$ 和 $A$ 的试验误差越趋于减小, 而 $m$ 的试验误差没有明显变化.

\subsection{8 湿度系统波动对试验误差的影响}

由于在实验过程中所设定的湿度值有一定波 动, 为考察湿度系统误差对试验误差的影响, 对每一 浓度点不叠加随机误差, 除每个湿度值分别叠加湿
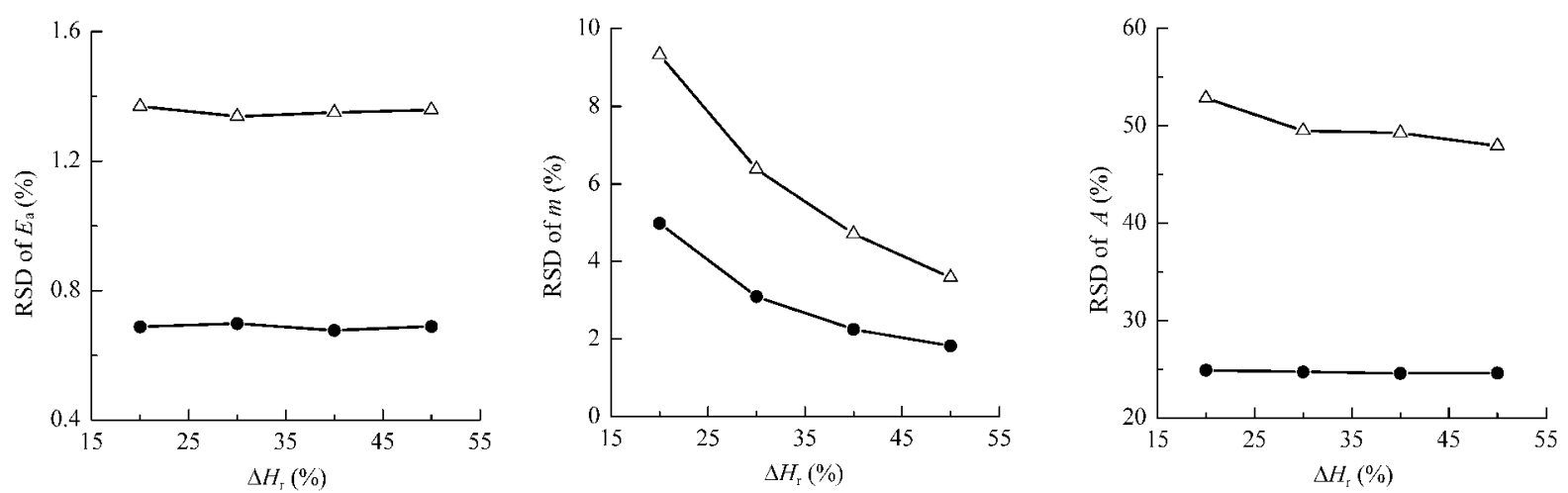

图 6 湿度差对动力学参数误差的影响

Fig.6 Effect of humidity change on kinetic parameter errors

data with $\pm 1 \%(\bullet)$ and $\pm 2 \%(\triangle)$ random error 

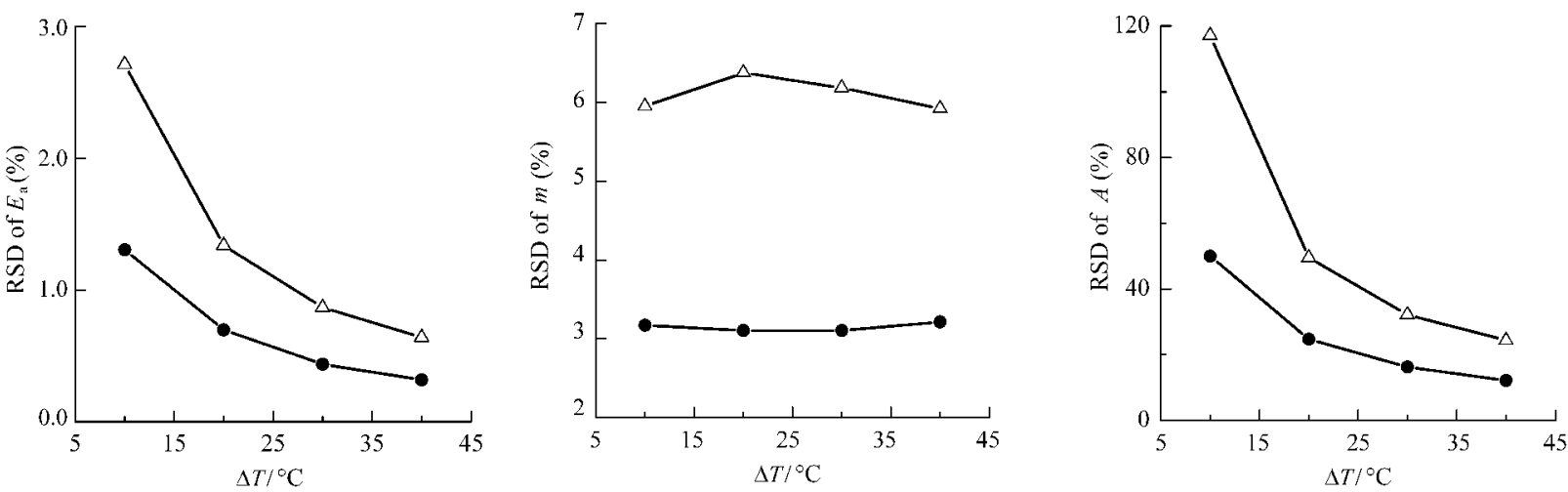

图 7 温度差对动力学参数误差的影响

Fig.7 Effect of temperature change on kinetic parameter errors

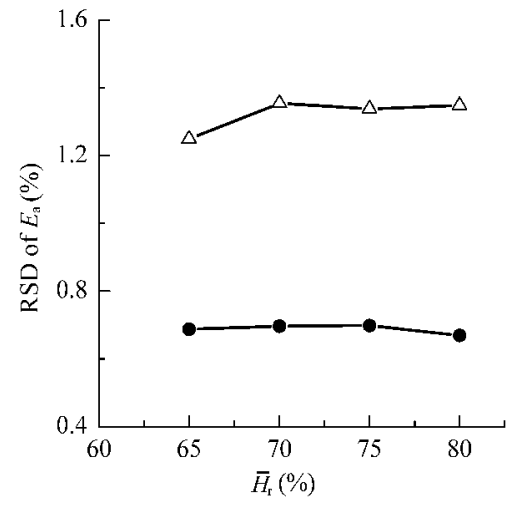

data with $\pm 1 \%(\bullet)$ and $\pm 2 \%(\triangle)$ random error
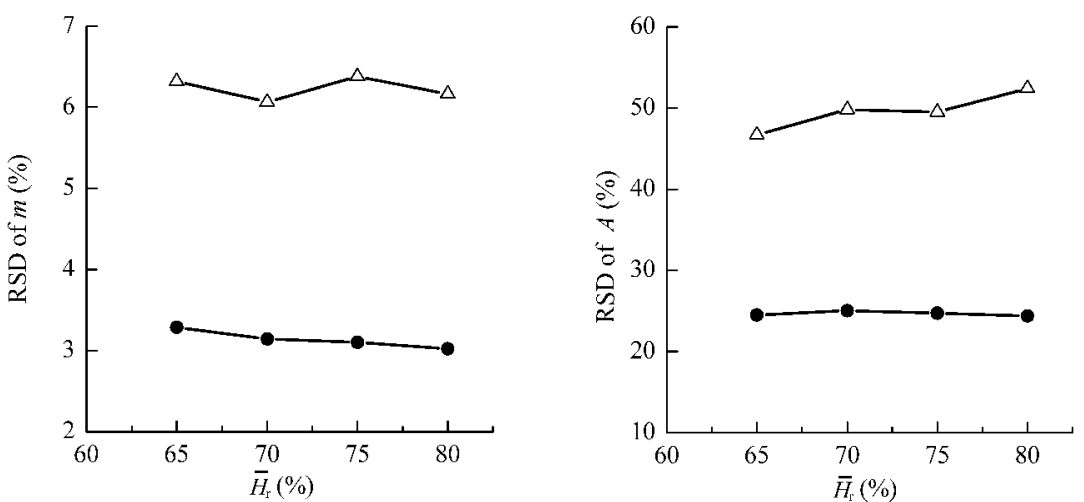

图 8 平均湿度 $\overline{\boldsymbol{H}}_{\mathrm{r}}$ 对动力学参数误差的影响

Fig.8 Effect of average humidity $\bar{H}_{\mathrm{r}}$ on kinetic parameter errors

data with $\pm 1 \%(\bullet)$ and $\pm 2 \%(\triangle)$ random error
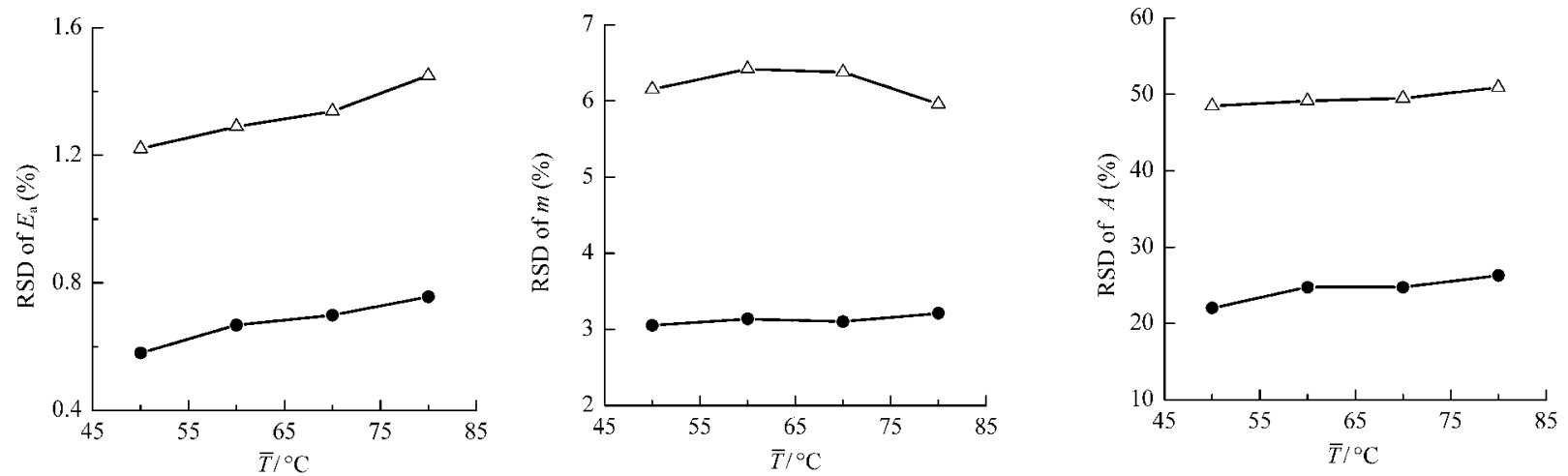

图 9 平均温度 $\bar{T}$ 对动力学参数误差的影响

Fig.9 Effect of average temperature $\overline{\boldsymbol{T}}$ on kinetic parameter errors

data with $\pm 1 \%(\bullet)$ and $\pm 2 \%(\triangle)$ random error

度系统误差 $\pm 0.3 \% 、 \pm 0.5 \% 、 \pm 0.8 \% 、 \pm 1.0 \%$, 含量均从 $100 \%$ 降解到 $80 \%$ 外, 其他条件及试验方法与 3.2 .1 节相同, 结果见图 10. 由图 10 可见, 随着湿度系统 误差的增大, $E_{\mathrm{a}} 、 m$ 和 $A$ 的试验误差也随之增大.

\section{2 .9 温度系统波动对试验误差的影响}

由于在实验过程中所设定的温度值有一定波
动, 为考察温度系统误差对试验误差的影响, 对每一 浓度点不叠加随机误差, 除每个温度值分别叠加温 度系统误差 $\pm 0.1 、 \pm 0.3 、 \pm 0.5 、 \pm 0.8{ }^{\circ} \mathrm{C}$, 含量均从 $100 \%$ 降解到 $80 \%$ 外, 其他条件及试验方法与 3.2 .1 节相 同, 结果见图 11. 由图 11 可见, 随着温度系统误差 的增大, $E_{\mathrm{a}} 、 m$ 和 $A$ 的试验误差也随之增大. 

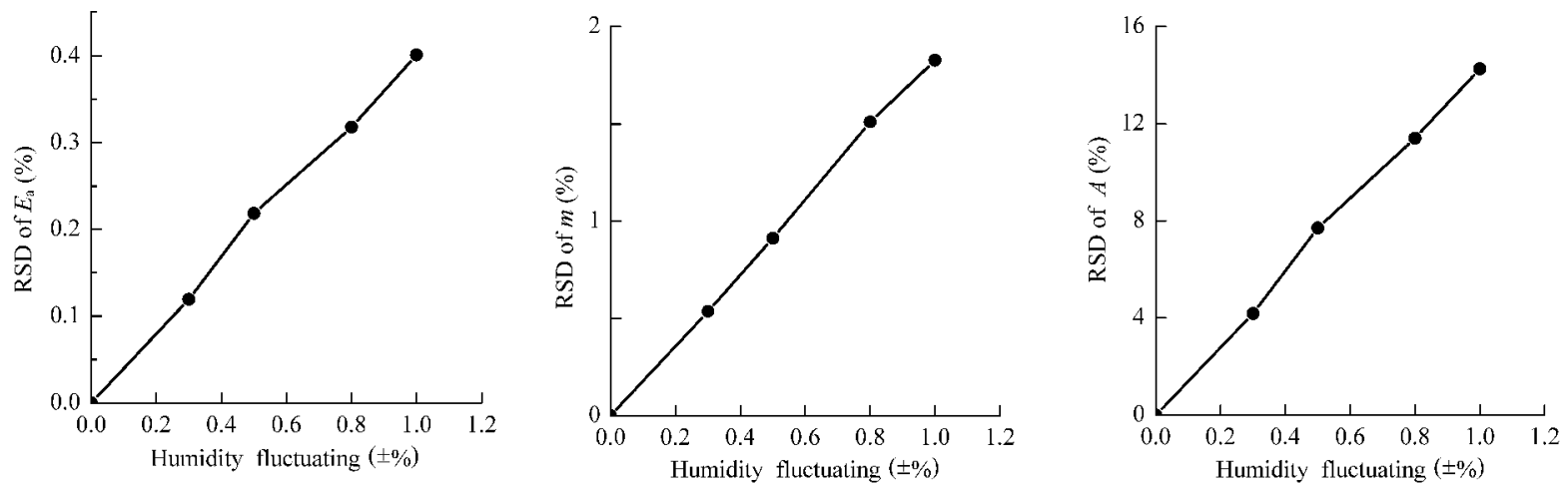

图 10 湿度系统波动对动力学参数误差的影响

Fig.10 Effect of humidity system fluctuating on kinetic parameter errors
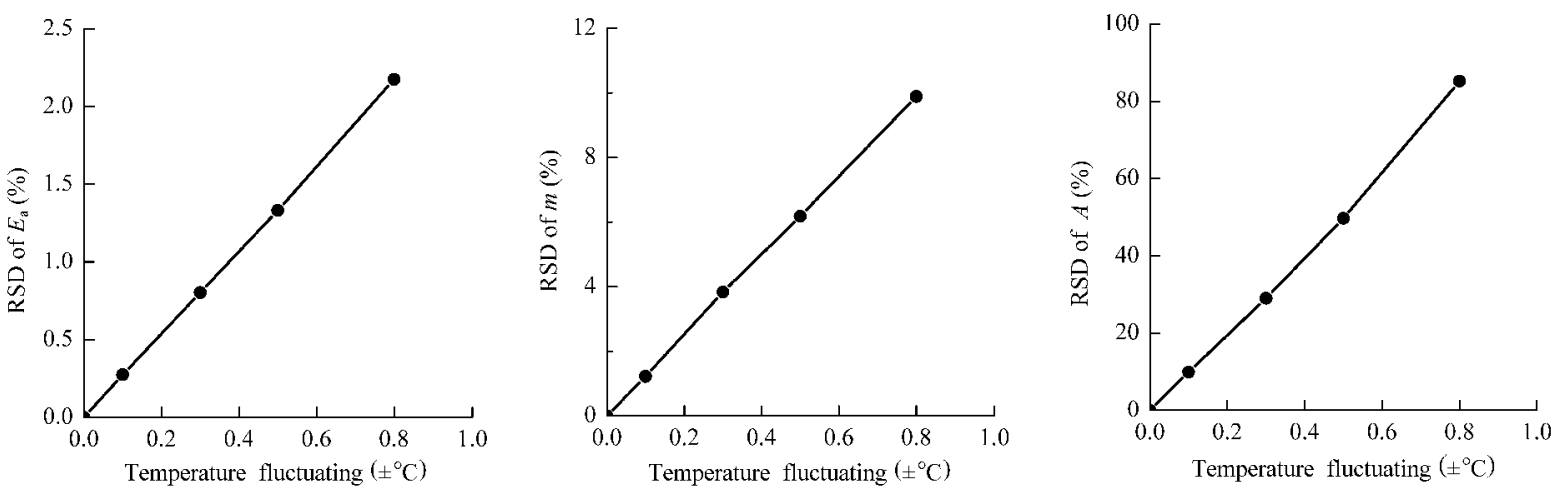

图 11 温度系统波动对动力学参数误差的影响

Fig.11 Effect of temperature system fluctuating on kinetic parameter errors

\section{4 结 论}

与经典恒温恒湿法和程序变温变湿法对比, 平 面单测点法采用均匀设计分散原理精确地确定了药 物的湿度、温度平面, 不仅实验工作量小、准确度和 精密度高, 而且只需使用普通的恒温恒湿控制装置. 通过计算机模拟固体阿司匹林的降解过程, 从理论 上考察了降解程度、抽样频率、湿度差、温度差、平均 湿度、平均温度、湿度波动和温度波动等不同试验条 件对平面单测点法测定误差的影响. 结果表明, 在平 面单测点法中, 通过增加药物的降解程度, 提高抽 样频率, 增大试验的湿度差和温度差, 使平均温度 接近室温, 控制湿度和温度系统误差, 都能减小试 验误差.

\section{References}

1 Leeson, L. J.; Mattocks, A. M. J. Am. Pharm. Assoc. Sci. Ed., 1958, 47: 329

2 Hasegawa, J.; Hanano, M.; Awazu, S. Chem. Pharm. Bull., 1975, 23(1): 86

3 Genton, D.; Kesselring, U. W. J. Pharm. Sci., 1977, 66(5): 676

4 Yoshioka, S.; Shibazaki, T.; Ejima, A. Chem. Pharm. Bull., 1982,
30(10): 3734

5 Yoshioka, S.; Uchiyama, M. J. Pharm. Sci., 1986, 75(5): 459

6 Carstensen, J. T.; Attarchi, F. J. Pharm. Sci., 1988, 77(4): 318

7 He, X. Y.; Yin, G. K.; Ma, B. Z. Acta Pharm. Sin., 1990, 25(7): 543 [何晓阳, 殷恭宽, 马宝忠. 药学学报, 1990, 25(7): 543]

8 Jelińska, A.; Zajac, M.; Gostomska, J. IL Farmaco., 2003, 58(4): 309

9 Zhao, Q.; Zhan, X. C.; Li, L. L. J. Pharm. Sci., 2005, 94(11): 2531

10 Yin, X. D.; Zhan, X. C.; Li, L. L.; Li, C. R.; Lin, T.; Zhao, Q.; He, N. Acta Chim. Sin., 2005, 63(6): 512 [尹小东, 詹先成, 李琳丽, 李成容, 林 涛, 赵 强, 何 宁. 化学学报, 2005, 63(6): 512]

11 Li, L. L.; Zhan, X. C.; Tao, J. L. Arch. Pharm. Res., 2008, 31(3): 381

12 Jiang, L; Zhan, X. C.; Li, L. L. West China J. Pharm. Sci., 2008, 23(1): 075 [蒋 露, 詹先成, 李琳丽. 华西药学杂志, 2008, 23 (1): 075]

13 Pang, Y. H.; Lu, C. S. J. Beijing Med. Coll., 1982, 14(3): 271 [庞贻慧, 鲁纯素. 北京医学院学报, 1982, 14(3): 271]

14 Ding, Q. L.; Mao, C. H. Chinese Traditional Patent Medicine, 1994, 16(6): 8 [丁青龙, 毛春红. 中成药, 1994, 16(6): 8]

15 Wang, Q. F.; Li, S. M.; Zhao, Z.; Zhu, Y. J. Shenyang Pharm. Univ., 1999, 16(3): 176 [王齐放, 李三鸣, 赵 喆, 朱 瑛. 沈阳 药科大学学报, 1999, 16(3): 176]

16 Fang, K. T. Uniform design and uniform design table. Beijing: 
Science Press, 1994: chapter 1 and 69-72 [方开泰. 均匀设计与 均匀设计表. 北京: 科学出版社, 1994: chapter 1 and 69-72]

17 Ban,Y. D.; Sun, Y. Q.; Mao, X. L. Acta Pharm. Sin., 1992, 27(10): 768 [班允东, 孙艈庆, 毛晓林. 药学学报, 1992, 27(10): 768]

18 Deng, Y. J.; Liu, S. Q.; Gu, X. Q. J. Shenyang Pharm. Univ., 1996, 13(1): 1 [邓英杰, 刘素琴, 顾学表. 沈阳药科大学学报, 1996, 13(1): 1]

19 Wen, W. Q.; Chen, B. J. Chinese Medicinal Materials, 1996, 19 (9): 474 [温文清, 陈 斌. 中药材, 1996, 19(9): 474]

20 Zhang, X. J.; Liu, M. S.; Chen, S. R. J. Shenyang Pharm. Univ., 1996, 13(4): 282 [张晓菁, 刘明生, 陈树人. 沈阳药科大学学报, 1996, 13(4): 282]
21 Lee, R. J.; Liu, Z.; Zou, H. F.; Ni, J. Y.; Zhang, Y. K. J. Microcolumn Separations, 2000, 12(6): 356

22 Cao, Y.; Xia, Q. R.; Fang, B. S. J. Chem. Technol. Biotechnol., 2006, 81: 109

23 Ding, H. B. F.; Douglas, D. R.; Edward, S.; Ming, T. Statist. Med., 2008, 27: 3071

24 Liu, W. Y. Pharmaceutical analysis. Beijing: People's Medical Publishing House, 2005: 129-130 [刘文英. 药物分析. 北京: 人民卫生出版社, 2005: 129-130]

25 National pharmacopoeia committee Ed. Chinese pharmacopoeia explanation. Beijing: Chemical Industry Press, 1993: 5-8 [国家药 典委员会编. 中国药典注释. 北京: 化学工业出版社, 1993: 5-8]

\section{第十五次全国电化学学术会议第一轮通知}

由中国化学会电化学委员会主办的第十五次全国电化学学术会议定于 2009 年 12 月 16-20 日在吉林省长春市吉林大学举行. 本次大会由 吉林大学承办, 中国科学院长春应用化学研究所协办. 本次会议的主题是电化学科学与技术.

全国电化学学术会议是国内规模化、系列化的学术会议之一, 每两年举办一次, 以此推动国内电化学领域的学科发展和科技进步, 促进科 研合作和技术转化. 这次会议是我国电化学界的又一次高水平、高信息容量的学术交流. 会议将构筑高水平的学术交流平台, 围绕电化学和技 术发展中的基础问题, 提出创新观点, 交流学术思想, 展示最新成果.

中国化学会电化学委员会热诚邀请大陆、港、澳、台地区和海外(华裔)从事电化学基础研究、应用研究及仪器开发的电化学家及产业界同 仁聚会长春, 交流和展示最新科研成果, 讨论电化学学科的前沿和基础问题, 探索如何进一步推动电化学科学和技术在国民经济发展中的应用 等问题.

\section{一、会议安排形式}

1、大会报告

(1) 有突出研究成果的电化学科学工作者的邀请报告,每人 30 分钟, 4 人左右; (2)经营优秀的企业界代表的邀请报告, 每人 20 分钟, 8 人左右.

\section{2 、主题报告}

取得优异研究成果的中、青年电化学科学工作者的邀请报告, 每人 30 分钟, 分 3 个会场, 每个分会场 8 人左右报告.

(1) 电化学基础研究; (2) 化学电源和超级电容器; (3) 其它研究方向.

3、分会报告

分 10 个分会场, 每人报告 15 分钟, 每个分会场 45 人左右报告.

(1) 电化学基础(包括谱学电化学和电催化)研究; (2) 锂离子电池; (3) 超级电容器与其它电池; (4) 燃料电池; (5) 工业电化学和电化学工程 (电解、电镀、金属腐蚀与防护、电沉积和表面处理技术); (6) 生物电化学; (7) 有机电化学和环境电化学; (8) 电分析化学和传感器; (9) 材料电化 学(纳米电化学、高功能金属和金属合金、高功能电化学聚合物、电极和电活性物质的分子设计); (10) 电化学设备与技术及其产业发展研讨会 (电解工业、电镀与修饰、湿法冶金、腐蚀与防护、电化学仪器和设备).

4、墙报

每篇论文限展一整张纸(一般高×宽= $110 \mathrm{~cm} \times 80 \mathrm{~cm}$ ), 同时, 在展出时间至少有一位作者现场讲解和回答问题.

5 、电化学技术及其产业发展专题讨论

6、产品及仪器展示

\section{二、投稿方式、起始和截止日期}

投稿起始和截止日期: 2009 年 6 月 30 日-9 月 30 日, 论文用在线投稿方式投稿, 论文格式要求及其它有关会议详情请登陆: http://09cse-online.org.

\section{三、联系方式}

地址: 长春市前进大街 2699 号吉林大学化学学院; 邮编: 130012; 联系人: 陆海彦, 黄卫民, 施峥;

电话:+86431-85155189; 传真: +86431-85155358; Email: luhy@jlu.edu.cn; huangwm@jlu.edu.cn; shizheng@jlu.edu.cn 